\title{
TREATMENT OF GENETIC DISORDERS
}

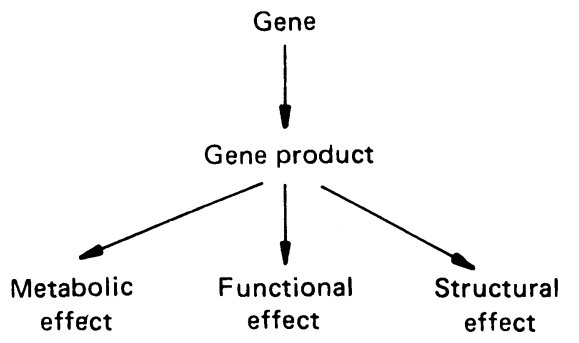

\section{Conventional treatment}

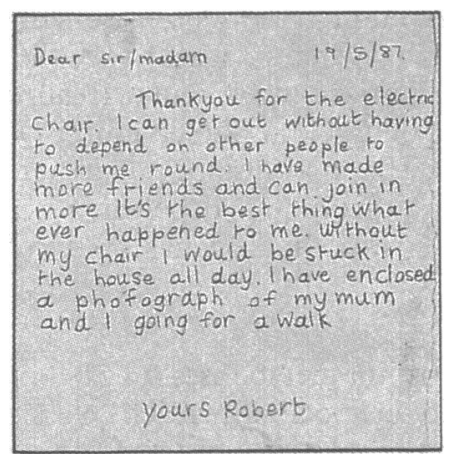

Letter written by boy aged 11 with Duchenne muscular dystrophy.

\section{Gene therapy}

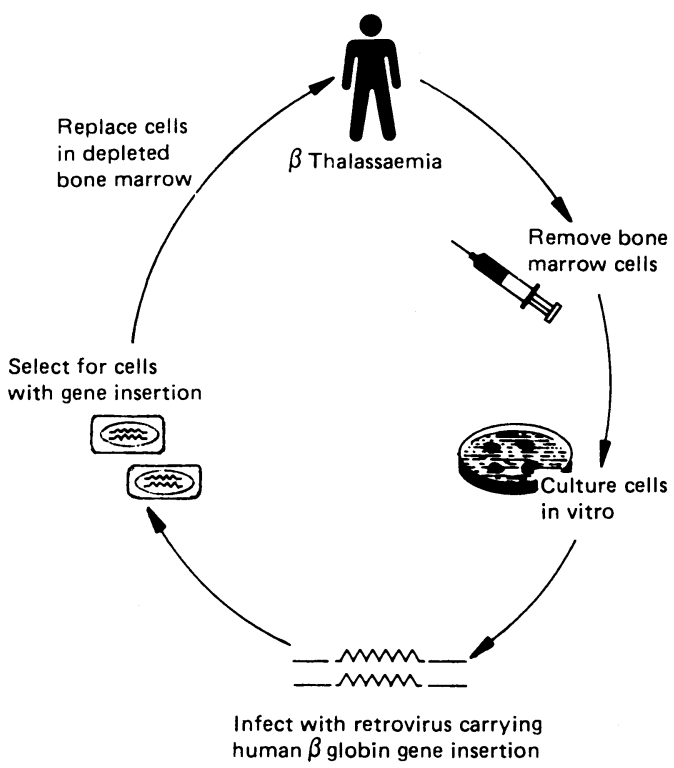

Potential strategy for gene therapy in $\beta$ thalassaemia.

\author{
Helen M Kingston
}

The prevention of inherited disease by means of genetic and reproductive counselling and prenatal diagnosis is often emphasised. Genetic disorders may, however, be amenable to treatment, either symptomatic or potentially curative. Treatment may range from conventional drug or dietary management and surgery to the future possibility of gene therapy. The level at which therapeutic intervention can be applied is influenced by the state of knowledge about the primary genetic defect, its effect, its interaction with environmental factors, and the way in which these may be modified.

Increasing knowledge of the molecular and biochemical basis of genetic disorders leads to better prospects for therapeutic intervention and even the possibility of prenatal treatment in some disorders. The primary defect in many disorders, however, is not yet amenable to specific treatment. Conventional treatment aimed at relieving the symptoms and preventing complications remains important and may require a multidisciplinary approach. Management of Duchenne muscular dystrophy, for example, includes neurological and orthopaedic assessment and treatment, physiotherapy, treatment of chest infections and heart failure, mobility aids, home modifications, appropriate schooling, and support for the family, all of which aim at lessening the burden of the disorder. Lay organisations often provide additional support for the patients and their families; the Muscular Dystrophy Group, for example, employs family care officers, who work closely with families and the medical services.

The prospect of curing genetic disorders with gene therapy is being investigated and has provoked much debate. In theory genetic disease could be cured by manipulating the genome directly to repair a genetic defect or by introducing a functioning donor gene into suitable recipient stem cells. Several experimental strategies have shown that gene transfer is possible. One method is to transfect host cells with-retroviruses carrying donor DNA, but this is not yet a therapeutic option in human disease. To be successful the donor DNA needs to be stably incorporated into the host genome and expressed in a controlled manner at adequate levels in the correct cells. Dangers of insertional mutagenesis exist; an inserted donor gene might disrupt a host gene and replace one genetic disorder with another or increase the risk of neoplasia. There are ethical concerns about manipulating the human genome and especially about introducing foreign DNA into germline cells. If a genetic manipulation is not heritable, however, affected descendants will also require treatment. 


\section{Gene product replacement}

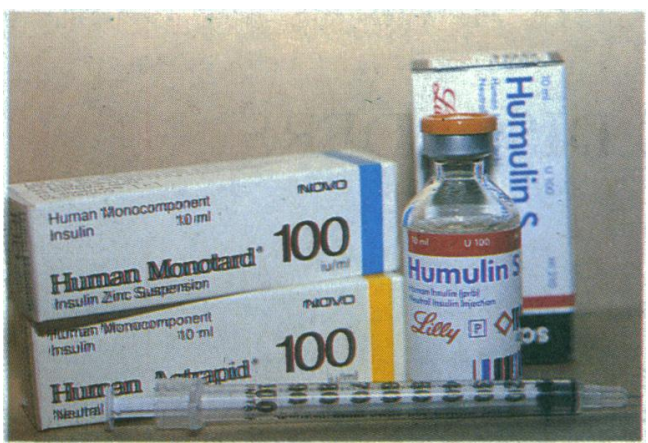

Insulins with human sequence prepared biosynthetically or by enzymatic modification of porcine material.
A simpler alternative to altering the genome is to replace a missing gene product. This strategy is effective when the gene product is a circulatory peptide or protein and is the standard treatment for haemophilia and growth hormone deficiency. The potential for direct replacement of missing intracellular enzymes is being determined experimentally. An alternative method of replacement is that of organ or cellular transplantation, which aims at providing a permanent functioning source of the missing gene product.

When the gene product is needed for metabolism within the central nervous system the blood-brain barrier presents an obstacle to systemic replacement treatment. Another potential problem is the initiation of an immunological reaction to the administered protein by the recipient. Successful production of human gene products, such as insulin and growth hormone, by recombinant DNA techniques may reduce this risk and will ensure adequate supplies for clinical use.

\section{Metabolic manipulation}

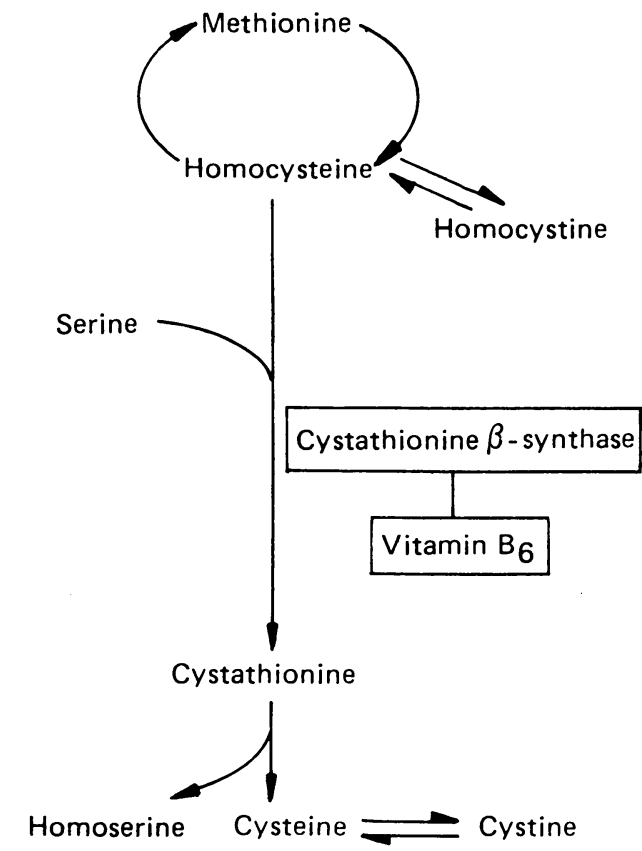

Pathway for homocysteine metabolism: most cases of homocystinuria are due to deficiency of cystathionine $\beta$-synthase, which requires vitamin $B_{6}$ cofactor.

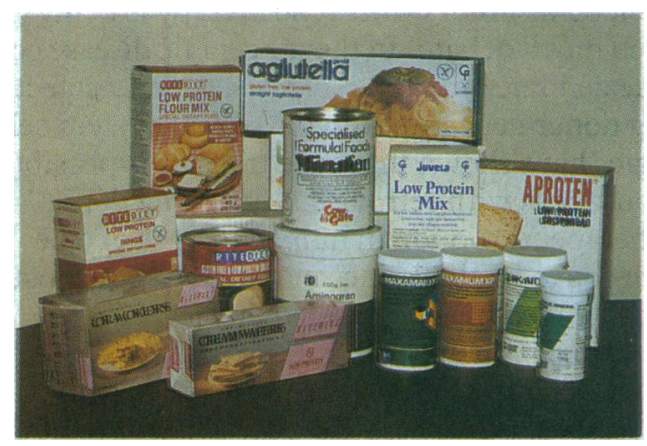

Products low in phenylalanine used in dietary management of phenylketonuria.
Many inborn errors of metabolism due to enzyme deficiencies can be treated effectively. Although direct replacement of the missing enzyme is not generally possible, enzyme activity can be enhanced in some disorders. For example, phenobarbitone induces hepatic glucuronyl transferase activity and may lower circulating concentrations of unconjugated bilirubin in the Crigler-Najjar syndrome type 2. Vitamins act as cofactors in certain enzymatic reactions and can be effective if given in doses above the usual physiological requirements; homocystinuria may respond to treatment with vitamin $B_{6}$, certain types of methylmalonic aciduria to vitamin $B_{12}$, and multiple carboxylase deficiency to biotin. It may also be possible to stimulate alternate metabolic pathways; thiamine may permit a switch to pyruvate metabolism by means of pyruvate dehydrogenase in pyruvate carboxylase deficiency.

The clinical features of an inborn error of metabolism may be due to accumulation of a substrate that cannot be metabolised. The classical example is phenylketonuria, in which the absence of phenylalanine hydroxylase results in high concentrations of phenylalanine, causing mental retardation, seizures, and eczema. The treatment consists of limiting dietary intake of phenylalanine to that essential for normal growth. Galactosaemia is similarly treated by a galactose free diet. In other disorders the harmful substrate may have to be removed by alternative means, such as the chelation of copper with penicillamine in Wilson's disease and peritoneal dialysis or haemodialysis in certain disorders of organic acid metabolism. In hyperuricaemia urate excretion may be enhanced by probenecid or its production inhibited by allopurinol, an inhibitor of xanthine oxidase.

In another group of inborn errors of metabolism the signs and symptoms are due to deficiency of an end product of a metabolic reaction, and treatment depends on replacing this end product. Defects occurring at different stages in biosynthesis of adrenocortical steroids in the various forms of congenital adrenal hyperplasia are treated by replacing cortisol, alone or together with aldosterone in the salt losing form. Congenital hypothyroidism can similarly be treated with thyroxine replacement. In some disorders, such as oculocutaneous albinism in which a deficiency in melanin production occurs, replacing the end product of the metabolic pathway is, however, not possible. 


\title{
Environmental modification
}

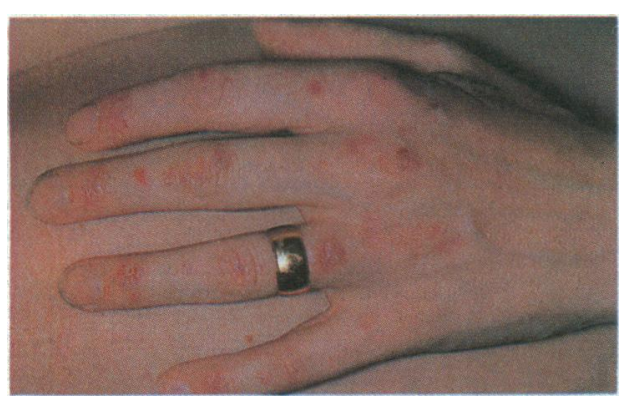

Porphyria cutanea tarda.

\section{Surgical management}

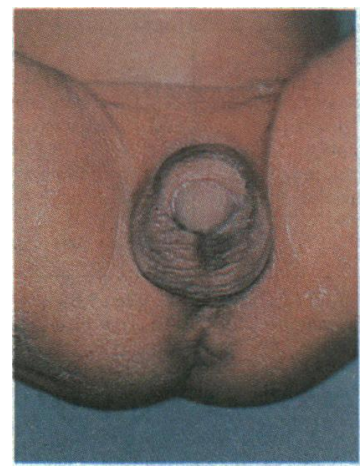

Virilisation of female genitalia in congenital adrenal hyperplasia (21-hydroxylase deficiency).
The effects of some genetic disorders may be minimised by avoiding known environmental triggers. Certain drugs will precipitate attacks in porphyria, including anticonvulsants, oestrogens, barbiturates, and sulphonamides in acute intermittent porphyria and oestrogens and alcohol in porphyria cutanea tarda. Other drugs, such as primaquine and dapsone, as well as ingesting fava beans cause haemolysis in glucose-6-phosphate dehydrogenase deficiency. Suxamethonium must not be given to people with pseudocholinesterase deficiency, and risks are associated with anaesthesia in myotonic dystrophy.

Exposure to sunlight precipitates skin fragility and blistering in all the porphyrias except the acute intermittent form. Sunlight should also be avoided in xeroderma pigmentosa (a rare defect of DNA repair) and in oculocutaneous albinism because of the increased risk of skin cancer.

Surgery plays an important part in various genetic disorders, not only those concerning primary congenital malformations. Virilisation of the external genitalia in girls with congenital adrenal hyperplasia is secondary to excess production of androgenic steroids in utero and requires reconstructive surgery. In some disorders structural complications may occur later, such as the aortic dilatation that may develop in Marfan's syndrome. Surgery may also be needed in genetic disorders that predispose to neoplasia, such as the multiple endocrine neoplasia syndromes, and screening family members at risk permits early intervention and improves prognosis. Occasionally malformations detected during pregnancy, such as posterior urethral valves, may be amenable to prenatal surgical intervention.

Dr Helen M Kingston, MD, is consultant clinical geneticist at St Mary's Hospital, Manchester.

The letter was reproduced by kind permission of Robert Little; the illustration of porphyria cutanea tarda by kind permission of Dr T Kingston, Skin Hospital, Salford; and that of congenital adrenal hyperplasia by kind permission of Dr Dian Donnai, St Mary's Hospital, Manchester.

The ABC of Child Abuse continues next week. The 13th article in this series will appear on 17 June.

\section{MATERIA PARAMEDICA}

\author{
Crisp and crunch
}

When we chew crisp food the bones of the skull perceive the vibrations emanating from the crunching, and they conduct the sound to our ears. This article explores the pleasurable aspects of these sensations, their causes and consequences.

There are not many naturally occurring foods that crunch when we chew them. Most of these are nuts and certain vegetables when eaten raw - for example, carrots, radishes, cauliflower, celery, and "iceberg" lettuce. Most crunchy foods acquire that property from pretreatment, and especially from removal of their water content. Thus simple drying, perhaps aided by heat, gives crispness to rusks, biscuits, and breakfast cereals. Frying likewise drives out the water that renders food structurally flexible, and if it is complete the food becomes rigid and brittle. Oil will then enter the interstices vacated by water but will fail to impart flexibility. This is well seen in potato crisps, and it occurs on the surface of freshly chipped potatoes. The list is endless. What is clear, however, is that crunching, up to a point, is enjoyable.

The manufacturers of sweets and candies have exploited the enjoyment derived from crunching in various ways. Fused sugar is brittle, especially when fat is melted in, as in butterscotch; it is then a useful medium for incorporating roasted nuts or sesame seeds. Chocolate is given a crunch by incorporation of nuts or of sugar fragments, often peppermint flavoured. Some manufacturers attract custom by using the word "crunchie" in the brand name of their product. But never mind the dental caries. Good parents will regulate consumption.

When our ancestors were hunter-gatherers, and no doubt even before that era, their main source of calcium after weaning would have been the bones of small animals. If there were any who failed to enjoy crunching small bones, they would probably have been eliminated by developing rickets and dying in obstructed parturition. Some years ago the lion cubs in the London Zoo developed rickets and fractures, and this was traced to their being fed on meat off the bone. When bones were included in the meal the disorder cleared up. I suggest that we may be descended from those ancestors who enjoyed crunching small bones and that we have retained this vestige - a vestige that now has diverted outlets. Crushing the bones of poultry and small animals with the teeth, however, is still practised by some Asians. ${ }^{1}$ - BERNARD J FREEDMAN

1 Kerrr NW. Diet and tooth wear. Scottish Med f 1988;33:313-5. 